\title{
Talcum Powder Toxicosis in Pregnancy
}

\author{
Jon F. Pennycuff, MD, MSPH ${ }^{1}$ Abigail Davenport, MD, MSM ${ }^{2}$ Jane Ellis, MD, $\mathrm{PhD}^{3}$ \\ Elizabeth Patberg, $\mathrm{MD}^{4}$ Carrie Cwiak, MD, $\mathrm{MPH}^{3}$
}
${ }^{1}$ National Center for Advanced Pelvic Surgery, Medstar Washington
Hospital Center, Georgetown University, Washington, D.C.
${ }^{2}$ Department of Obstetrics and Gynecology, MetroHealth Medical Center, Cleveland, Ohio
${ }^{3}$ Department of Gynecology and Obstetrics, Emory University School of Medicine, Atlanta, Georgia
${ }^{4}$ Department of Obstetrics and Gynecology, University of California, Irvine Medical Center, Orange, California

Address for correspondence Elizabeth Patberg, MD, Department of Obstetrics and Gynecology, University of California, Irvine Medical Center, 333 City Blvd West, Ste 1400, Orange, CA 92868 (e-mail: epatber@emory.edu).

Am J Perinatol Rep 2018;8:e384-e386.

\begin{abstract}
Background Pica is a relatively common phenomenon in pregnancy and typically includes consumption of nontoxic substances such as earth/clay, raw starches, and ice. Occasionally, substances may be toxic or have unintended consequences.

Case A nulliparous woman presented to our facility complaining of numerous, vague symptoms that are common in pregnancy. She had multiple work-ups and an admission to our antepartum unit without clear etiology of her symptoms. Ultimately, she was

Keywords

- talcum

- toxicosis

- pregnancy

- pica diagnosed with talcum powder toxicosis secondary to talc ingestion as a coping mechanism for her anxiety, which was heightened in pregnancy.

Conclusion This case highlights the importance of screening for mental health disorders, which may be exacerbated during the peripartum period. Patients' coping mechanisms for mental health disorders may have unintended consequences.
\end{abstract}

Pica is the purposeful ingestion of nonfood substances. The International Classification of Diseases, Tenth Revision defines pica as "the tendency or craving to eat substances other than normal foodstuffs." 1 Pica is a known phenomenon in pregnancy, with a reported incidence that varies widely from 0.02 to $38 \%$ and may be related to demographic variables including race and socioeconomic status. ${ }^{2-4}$ The most common substances ingested include earth/clay (geophagy), raw starches (amylophagy), and ice (pagophagy). In rare instances, dangerous substances such as glass, lighters, or needles may be consumed. ${ }^{5}$

Multiple theories have been proposed to explain why pregnant women consume nonfood substances, and the disorder is likely multifactorial. These hypotheses include cultural expectations, psychological stress, hunger, dyspepsia, micronutrient deficiencies, and intended protection against toxins and pathogens-all of which can increase during pregnancy. ${ }^{5}$ While pica is generally benign, the excessive ingestion of certain nonfood substances has the potential to cause maternal toxicity, fetal harm, or teratogenicity.

received

April 22, 2017

accepted

September 8, 2018
DOI https://doi.org/

$10.1055 / \mathrm{s}-0038-1676382$. ISSN 2157-6998.
Here, we report a case of a woman whose pica in pregnancy manifested as multiple, nonspecific complaints and led to a delay in her diagnosis.

\section{Case}

A 18-year-old multiracial G2P0010 presented to our facility at 24 weeks' estimated gestational age complaining of loss of fluid. At that time, she also endorsed abdominal pain, headache, diffuse myalgias, rhinorrhea, oropharyngeal irritation, ear pain, cough, intermittent shortness of breath, and palpitations coinciding with anxiety. Her first pregnancy resulted in an early pregnancy loss necessitating a dilation and curettage. She had been receiving routine prenatal care during this pregnancy. Her past medical history was significant for anxiety. She had recently emigrated from Canada and was living with her boyfriend. She had a preexisting history of heart palpitations, but her prior work-up was found to be negative. This pregnancy was notable for a prior triage visit for shortness of breath at

Copyright $\odot 2018$ by Thieme Medical Publishers, Inc., 333 Seventh Avenue, New York, NY 10001, USA. Tel: +1(212) 584-4662.
License terms

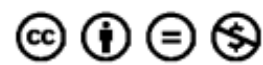


19 weeks at which time she was diagnosed with physiological respiratory change of pregnancy.

At the time of her triage visit at 24 weeks, she was febrile to $38^{\circ} \mathrm{C}$, with a pulse of 107 , blood pressure of $99 / 61$, and $\mathrm{O}_{2}$ saturation of $100 \%$. She ruled out for rupture of membranes. Her examination was positive for left-sided costovertebral tenderness, although her urinalysis was not consistent with urinary tract infection. Nevertheless, due to her symptoms and exam, she was admitted to our facility's antepartum unit and was treated empirically with antibiotics for pyelonephritis. When her fever resolved, she was discharged and was eventually found to have a negative urine culture.

The patient returned to our facility at 25.5 weeks' estimated gestational age complaining of subjective fevers, diarrhea, night sweats, cough, oropharyngeal irritation with mucus discharge from her nose, and shortness of breath. She had experienced constipation and right-sided abdominal pain, which had resolved by the time of presentation. There was concern for infectious etiology. She denied sick contacts, did not endorse recent travel, and had no risk factors for tuberculosis. She was again noted to be febrile to $38^{\circ} \mathrm{C}$ but was otherwise vitally stable, with $\mathrm{O}_{2}$ saturation of $99 \%$ on room air. She had a benign physical examination. Chest X-ray was unremarkable. Her hemoglobin was $12.6 \mathrm{~g} / \mathrm{dL}$, with hemoglobin AA by electrophoresis. White blood cell count was 12,600 cells per cubic millimeter. Chemistry panel and thyroid stimulating hormone were within normal limits. A purified protein derivative (PPD) test was placed at this visit and was subsequently found to be negative. A stool sample was requested for analysis of ova and parasites, but the patient failed to provide a feces sample.

During subsequent questioning, the patient endorsed chronic ingestion of "baby powder." She stated that she had been eating baby powder since adolescence but had increased her intake since becoming pregnant. The patient reported that this had been a coping mechanism for her anxiety, which had acutely worsened during this pregnancy. She then provided a bottle of baby powder, which she had hidden in her bed during her hospital admission. She noted that the symptoms began with her increased intake of the baby powder. The Toxicology service was consulted. They confirmed her symptoms were consistent with talc toxicity, most notably cough, oropharyngeal irritation, diarrhea, vomiting, low blood pressure, chest pain, cough, shortness of breath, and fever. She was counseled of the risks to herself and to her fetus. A referral to the outpatient Obstetric Psychiatry service was made, and the patient was discharged to home when her fever and symptoms resolved.

The patient attended multiple behavioral health sessions and noted improvement in her anxiety symptoms. She was able to nearly abstain from talcum powder ingestions, noting only one relapse. The remainder of her antepartum care was unremarkable until she was admitted at 35.2 weeks for preterm premature rupture of membranes. She underwent induction of labor with oxytocin. She progressed to a normal spontaneous delivery of a viable, 2,915-g male infant with Apgar scores of 8 and 9 at 1 and 5 minutes, respectively. She had an uncomplicated postpartum course. The patient and her infant were discharged on postpartum day 3 . The patient returned 5 weeks postpartum for routine visit. She was doing well. She had discontinued mental health services as she no longer felt symptomatic and did not think she would benefit from behavioral health services. She opted for condoms and plan B for her postpartum contraceptive method.

\section{Discussion}

Talc is hydrated magnesium silicate $\left(\mathrm{Mg}_{3} \mathrm{Si}_{4} \mathrm{O}_{10}(\mathrm{OH})\right)$ and is a naturally occurring mineral found throughout the world. Talc was introduced in 1893 for use as baby powder. Its innate characteristics such as softness, hydrophobicity, organophilicity, and inertness make it an ideal anticaking agent, carrier, thickener, or absorbent filler., 6 Today, talc is used across multiple industrial and consumer products including food, paper, ceramics, cosmetics, and pharmaceuticals, among others. ${ }^{6-8}$ Exposure to large quantities of talc often occurs in persons working in these industries. Nonindustrial workers are typically exposed to talc through food, cosmetics, and pharmaceutical products. Although talc-containing products are widely used, data regarding its toxicity appear to be lacking.

Within the food industry, talc is most commonly used in the coating of gum and rice. ${ }^{9}$ According to the U.S. Food and Drug Administration, talc consumption per capita in rice products is approximately $0.5 \mathrm{~g}$ per day. ${ }^{9}$ Existing evidence does not suggest that talc is toxic at these levels. ${ }^{7}$ However, few studies documenting the toxicity of ingested talc exist. The Toxicology Data Network describes talc as "practically nontoxic" with a probable oral lethal dose above $15 \mathrm{~g} / \mathrm{kg}^{10}$ While ingestion of talc during pregnancy has not been studied extensively in humans, rat and rabbit models have shown no adverse fetal effects when exposed to oral talc. ${ }^{7,10}$ Nonetheless, the National Library of Medicine states that talcum powder toxicosis may occur when a person accidentally or intentionally inhales or ingests talcum powder. ${ }^{11}$

Talcum toxicosis is most commonly caused by inhalational exposure. Symptoms are nonspecific and affect many organ systems. Those affected may experience decreased urine output, cough, eye irritation, oropharyngeal irritation, diarrhea, vomiting, cardiovascular collapse, hypotension, seizures, chest pain, cough, difficulty breathing, respiratory failure, rapid and shallow breathing, wheezing, altered mental status, fever, lethargy, muscle twitching, blisters, and cyanosis. ${ }^{11}$ Inhalation of talc has been established as a cause of pneumoconiosis in mine workers or those exposed to large quantities of inhaled talc in other industries. Acute exposure may cause acute lung injury, respiratory failure, pneumonitis, or pneumonia. ${ }^{12}$ Chronic exposure to talc results in lung fibrosis and granuloma development. ${ }^{7}$ Although talc inhalation was once thought to contribute to the development of lung cancer, this has since been disproven, and limited studies exist regarding the overall carcinogenicity of talc. $^{6,7}$ Due to the paucity of data, talc that does not contain asbestos is "not classifiable as to carcinogenicity in humans" according to the International Agency for Research on Cancer. ${ }^{6}$

Pica in pregnancy is a well-documented phenomenon that has been occurring since antiquity. A meta-analysis by Fawcett 
et al found a $27.8 \%$ incidence of pica in pregnancy and the postpartum period; however, they noted that there is substantial heterogeneity both regionally and globally. ${ }^{14}$ In the United States, pica is more common among women living in the South. ${ }^{13}$ Pica has been associated with lower socioeconomic status, lower educational attainment, anemia, developmental disabilities, and African-American ethnicity. 4,13,14 The degree of harm to the patient depends on the type of substance being ingested, and testing should be based on the type of pica behavior. Severe lead poisoning including congenital lead poisoning has been described. ${ }^{15}$ Heavy metal poisoning with mercury and arsenic may also occur. ${ }^{13}$ Pica may also lead to gastrointestinal blockages, bowel perforation, or intestinal ischemia. ${ }^{13}$

Mishori and McHale recommend that high-risk groups including pregnant women, immigrants or refugees, and children or adults with autism or developmental disabilities be screened for pica behavior or unusual cravings. ${ }^{13}$ As pica is associated with anemia, a serum hemoglobin and hematocrit should be obtained in addition to iron studies. Testing for other nutrients and electrolytes including potassium, zinc, and calcium may also be warranted. Additionally, testing for lead, mercury, or arsenic may be indicated. $4,13,15$

Behavioral therapy is the mainstay of treatment for pica, with a treatment response rate of up to $80 \%{ }^{16}$ Successful treatment with selective serotonin reuptake inhibitors, atypical neuroleptics, and attention deficit/hyperactivity disorder medications has also been documented. ${ }^{13}$ Thus, referral to behavioral therapy and psychiatric services may be warranted in some patients. Any underlying anemia should be treated with iron supplementation. Abnormalities in electrolytes and micronutrients may also require supplementation. Heavy metal poisoning may require chelation therapy. ${ }^{15}$

In addition to routine prenatal care, at our institution, we recommend that consideration be given to implementing antenatal testing in a patient such as the one described in this case report. The frequency and methods should be individualized in accordance with the patient's situation. For a patient such as ours, periodic fetal growth scans every 3 to 4 weeks and a weekly biophysical profile initiated at 32 to 34 weeks and continued until delivery would allow increased surveillance of both the mother and the fetus. If maternal and fetal status remains reassuring, delivery at 39 to 40 weeks would be reasonable unless indications arose for earlier delivery.

\section{Conclusion}

We described a case of unintentional talcum toxicosis in a pregnant patient. The patient's multiple, vague symptoms were likely due to chronic, small dose inhalation of aerosolized talc during ingestion. The gastrointestinal symptoms were likely due to the irritating and desiccating properties of talc. The fact that the patient's symptoms were nonspecific, and included some symptoms common in pregnancy, contributed to her delayed diagnosis and multiple triage visits in the interim. This case highlights the importance of screening for mental health disorders, which may be exacerbated during the peripartum period. Patients' coping mechanisms for these mental health disorders may have unintended consequences. Including an exacerbation of her mental health disorder in the differential, followed by careful history-taking, led to the correct diagnosis and treatment.

\section{Conflict of Interest}

None.

\section{References}

1 World Health Organization; 1992The ICD-10 Classification of Mental Health and Behavioural Disorders: Clinical Descriptions and Diagnostic Guidelines. Geneva: World Health Organization

2 Corbett RW, Ryan C, Weinrich SP. Pica in pregnancy: does it affect pregnancy outcomes? MCN Am J Matern Child Nurs 2003;28(03): 183-189, quiz 190-191

3 Mikkelsen TB, Andersen AM, Olsen SF. Pica in pregnancy in a privileged population: myth or reality. Acta Obstet Gynecol Scand 2006;85(10):1265-1266

4 Cardwell MS. Eating disorders during pregnancy. Obstet Gynecol Surv 2013;68(04):312-323

5 Young SL. Pica in pregnancy: new ideas about an old condition. Annu Rev Nutr 2010;30:403-422

6 International Agency for Research on Cancer, Carbon black, titanium dioxide and talc. IARC Working Group on the Evaluation of Carcinogenic Risks to Humans: IARC Monographs on the Evaluation of Carcinogenic Risks to Humans: Carbon black, titanium dioxide, and talc, 93. 2010. http://monographs.iarc.fr/wp-content/uploads/ 2018/06/mono93.pdf. Accessed December 19, 2018

7 Harbison RD, Bourgeois MM, Johnson GT. Hamilton and Hardy's Industrial Toxicology. Hoboken, NJ: John Wiley \& Sons Inc.; 2015

8 Garlich FM, Nelson LS. Inhalation of baby powder. Emed Mag; 2011:17-20

9 FDA GRAS substances. U.S. Food and Drug Administration. Select Committee on GRAS Substances (SCOGS) Opinion: Silicates. http://wayback.archive-it.org/7993/20171031063508/https:// www.fda.gov/Food/IngredientsPackagingLabeling/GRAS/SCOGS/ ucm260849.htm. Accessed December 19, 2018

10 Toxnet. U.S. National Library of Medicine. Toxnet: Toxicology Data Network: HSDB: Magnesium Silicate. http://toxnet.nlm.nih.gov/ cgi-bin/sis/search2/f?/temp/ jYLj3w:3. Accessed July 28, 2015

11 Talcum Powder Poisoning. Medline Plus. U.S. National Library of Medicine. http://www.nlm.nih.gov/medlineplus/ency/article/00 2719.htm. Accessed July 29, 2015

12 Lawson R. Acute talc inhalation. Nursing 2012;42(01):72

13 Mishori R, McHale C. Pica: an age-old eating disorder that's often missed. J Fam Pract 2014;63(07):E1-E4

14 Fawcett EJ, Fawcett JM, Mazmanian D. A meta-analysis of the worldwide prevalence of pica during pregnancy and the postpartum period. Int J Gynaecol Obstet 2016;133(03):277-283

15 Shannon M. Severe lead poisoning in pregnancy. Ambul Pediatr 2003;3(01):37-39

16 Hagopian LP, Rooker GW, Rolider NU. Identifying empirically supported treatments for pica in individuals with intellectual disabilities. Res Dev Disabil 2011;32(06):2114-2120 